\title{
Multimodal brain imaging connectivity analyses of emotional and motivational deficits in depression among women
}

\author{
Gabriel Robert, MD, PhD; Elise Bannier, PhD; Magali Comte, PhD; Lea Domain, MD; \\ Isabelle Corouge, PhD; Thibaut Dondaine, PhD; Jean-Marie Batail, MD, PhD; \\ Jean-Christophe Ferre, MD, PhD; Eric Fakra, MD, PhD; Dominique Drapier, MD, PhD
}

\begin{abstract}
Background: Major depressive disorder (MDD) is characterized by impaired cortical-subcortical functional connectivity. Apathy adds to functional impairment, but its cerebral basis in MDD remains unknown. Our objective was to describe impairments in functional connectivity during emotional processing in MDD (with varying levels of congruency and attention), and to determine their correlation with apathy. Methods: We used the Variable Attention Affective Task during functional MRI, followed by diffusion-weighted MRI, to assess 55 right-handed women (30 with MDD and 25 healthy controls) between September 2012 and February 2015. We estimated functional connectivity using generalized psychophysiologic interaction and anatomic connectivity with tract-based spatial statistics. We measured apathy using the Apathy Evaluation Scale. Results: We found decreased functional connectivity between the left amygdala and the left anterior cingulate cortex (ACC) during negative stimuli in participants with $\mathrm{MDD}\left(t_{54}=4.2 ; p=0.035\right.$, family-wise error [FWE]-corrected). During high-attention stimuli, participants with MDD showed reduced functional connectivity between the right dorsolateral prefrontal cortex (dIPFC) and the right ACC $\left(t_{54}=4.06, p_{\mathrm{FWE}}=0.02\right)$, but greater functional connectivity between the right dIPFC and the right amygdala $\left(t_{54}=3.35, p=0.048\right)$. Apathy was associated with increased functional connectivity between the right dIPFC and the right ACC during high-attention stimuli $\left(t_{28}=5.2, p=0.01\right)$ and increased fractional anisotropy in the right posterior cerebellum, the anterior and posterior cingulum and the bilateral internal capsule (all $p_{\mathrm{FWE}}<0.05$ ). Limitations: Limitations included a moderate sample size, concomitant antidepressant therapy and no directed connectivity. Conclusion: We found that MDD was associated with impairments in cortical-subcortical functional connectivity during negative stimuli that might alter the recruitment of networks engaged in attention. Apathy-related features suggested networks similar to those observed in degenerative disorders, but possible different mechanisms.
\end{abstract}

\section{Introduction}

A categorical classification of mental disorders describes major depressive disorder (MDD) as a mood and/or interestrelated disorder, ${ }^{1}$ but MDD includes impaired emotional regulation and reduced motivation. ${ }^{2}$ Emotional disturbances include heightened responsiveness to negative events at the behavioural, cognitive and cerebral levels, ${ }^{3}$ underpinned by abnormal cortical-subcortical (top-down) regulation. ${ }^{4}$ More precisely, impaired control from cortical (top) structures (such as the dorsolateral prefrontal cortex $[\mathrm{dlPFC}]^{5}$ and the anterior cingulate cortex $[\mathrm{ACC}]^{6,7}$ ) over subcortical (down) structures (the amygdala) are thought to be responsible for negative emotions in MDD. ${ }^{4}$ Furthermore, reduced perigenual-amygdalar functional connectivity is supported by serotonin transporter polymorphism in healthy individuals ${ }^{8}$ and might be an intermediate phenotype of MDD. ${ }^{9}$ These key cortical-subcortical interactions have been assessed using emotional labelling tasks, but conflictual and high-attention stimuli are ubiquitous. We do not yet know how cortical-subcortical functional networks are impaired in MDD in contexts of varying attention and congruency.

Depression is characterized by emotional deficits, but also by motivational impairments, such as apathy. ${ }^{10}$ Apathy is defined as a reduction in goal-directed activities, including those in the cognitive, emotional and social dimensions. ${ }^{11}$ Motivational disorders increase the risk of recurrence, and they are associated with poorer $\operatorname{cognitive} \mathrm{e}^{10}$ and general ${ }^{12}$ function. Research domain criteria describe motivational deficits across many psychiatric disorders, including MDD. ${ }^{2}$

Cerebral bases for apathy have been characterized in degenerative disorders and point to frontal cortical-subcortical

Correspondence to: G.H. Robert, Pôle Hospitalo-Universitaire de Psychiatrie Adulte, Centre Hospitalier Guillaume Régnier, 35000 Rennes, France; gabriel.hadrien.robert@gmail.com

Submitted Apr. 15, 2020; Revised Jul. 28, 2020; Revised Oct. 25, 2020; Accepted Nov. 1, 2020

DOI: $10.1503 /$ jpn.200074 
networks, including the superior frontal gyri, the anterior cingulate and the ventral striatum in both Alzheimer and Parkinson disease. ${ }^{13,14}$ In healthy individuals, motivation is supported by functional connectivity in the dlPFC-ACCventral striatum and amygdala. ${ }^{15}$ This suggests that both emotion and motivation engage functional connectivity between the dIPFC and ACC and subcortical structures such as the amygdala or ventral striatum, implying common cerebral networks. We have shown that motivation and emotion deficits are correlated in Parkinson disease ${ }^{16}$ and in schizophrenia, ${ }^{17}$ but whether this is the case in MDD remains unknown.

To investigate the functional connectivity involved in emotion during various attentional and conflictual situations, we carried out a multimodal study using functional and structural MRI in women with MDD compared to healthy controls. During functional MRI, we used the Variable Affective and Attention Task (VAAT), ${ }^{18-20}$ designed to characterize the cerebral correlates that underlie emotional labelling according to 3 different parameters: valence (negative versus positive), congruency (incongruent versus congruent) and attention (high attention versus low attention). Variation of these parameters promotes the activity and connectivity of cerebral structures involved in emotional processing (i.e., the amygdala for valence, the dIPFC for attention and the ACC for congruency). The VAAT relies on an a priori anatomo-functional model of emotion regulation in which the dIPFC, ACC and amygdala interact through top-down and bottom-up regulatory mechanisms. ${ }^{18-21}$ We tested for apathy-related activation and functional connectivity. Indeed, the dlPFC, ACC and amygdala are the 3 main structures engaged in apathy. Based on findings from the early stages of degenerative disorders that showed enhanced functional connectivity between frontal areas associated with apathy, ${ }^{22,23}$ we expected to find enhanced functional connectivity between the dIPFC and the ACC.

We also used whole-brain voxel-wise tract-based spatial statistics $^{24}$ to test for differences in structural connectivity between groups. We expected our findings to be consistent with those from meta-analyses of voxel-wise fractional anisotropy in MDD, which found decreased fractional anisotropy in various tracts but most frequently in the anterior cingulum, the uncinate and the forceps minor. ${ }^{25-27}$ Similarly, we expected to find apathy-related anatomic connectivity features in the ACC based on findings from recent literature. ${ }^{28,29}$

\section{Methods}

\section{Participants}

All participants provided written informed consent. The study received approval from the local institutional review board (DEP-APATHIE 12/07-840; May 9, 2012).

\section{MDD participants}

The MDD group included 30 women who were right-handed (assessed using the Edinburgh Laterality Questionnaire) and had unipolar depression (assessed using the Mini International Neuropsychiatric Interview). We recruited women to homo- genize the population and maximize our chances of recruitment, because the sex ratio for MDD is 1.7:1 (women:men). ${ }^{30}$ Exclusion criteria for the MDD group were as follows: MRI contraindication (metallic implant, stroke, traumatic brain injury); severe cognitive disorder (Mattis Dementia Rating Scale [MDRS] < 130); high risk of suicide (Clinical Global Impression-Severity of Suicidality scale $>3$ ); or DSM-IV Axis 1 comorbidity (including bipolar disorder type 1 or type 2, and anxiety disorders). All patients were taking medication, except for 4 receiving neuromodulation ( 2 were receiving maintenance electroconvulsive therapy once a month), 1 receiving direct transcranial stimulation and 1 receiving repetitive transcranial magnetic stimulation (Appendix 1, Table S1, available at jpn.ca/200074-a1). At least 4 weeks had elapsed between the last neuromodulation session and the assessment.

\section{Healthy controls}

The healthy control group included 25 women who were right-handed (assessed using the Edinburgh Laterality Questionnaire). Exclusion criteria were as follows: any psychiatric disorder; MRI contraindication; stroke; traumatic brain injury; severe cognitive disorder (MDRS < 130), high risk of suicide (Clinical Global Impression-Severity of Suicidality scale $>3$ ). Healthy controls received $€ 50$ for participation.

\section{Clinical assessment}

We assessed depression severity using the MontgomeryÅsberg Depression Rating Scale (MADRS). ${ }^{31}$ We assessed apathy using the Apathy Evaluation Scale (AES). ${ }^{32}$

\section{Cognitive measures}

We assessed general cognitive function using the MDRS and executive function using the Stroop Colour and Word Test, the Trail-Making Test, the Modified Wisconsin Card Sorting Test (MCST), the digit symbol subtest of the Weschler Adult Intelligence Scale and tests of phonemic, semantic and action verb verbal fluency.

\section{VAAT}

During functional MRI, participants viewed images composed of 2 parts. The central part displayed discrete facial emotions - positive (joy) or negative (fear, disgust or anger). The central parts were superimposed on background scenes of complex emotion. Each emotional background scene was either positive or negative, sampled from the International Affective Picture System files. ${ }^{33}$ Either the central part or the background was highlighted using a green frame (block-wise). Participants labelled the part of the image framed in green as either pleasant or unpleasant (Figure 1). The VAAT uses a mixed eventrelated/block design and a factorial model that has 3 conditions (valence, congruency and attention), each with 2 possible levels: valence could be positive or negative; congruency could be congruent (the 2 parts of the image had similar emotional content) or incongruent (the 2 parts of the image had opposite emotional content); and attention load could be high 
(A) Valence

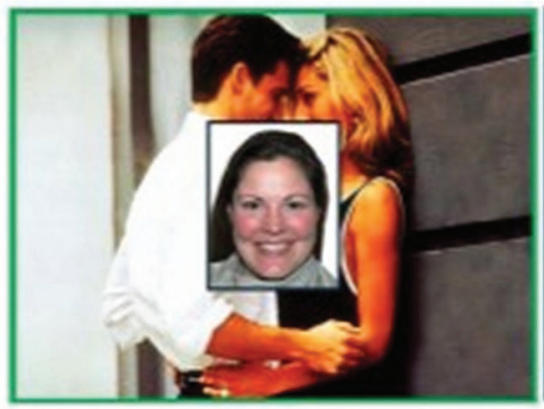

(1) Positive

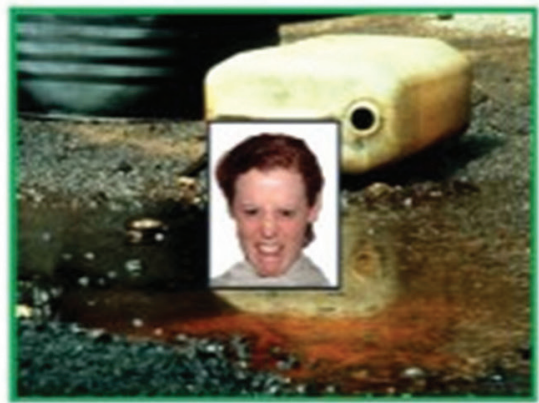

(2) Negative
(B) Congruency

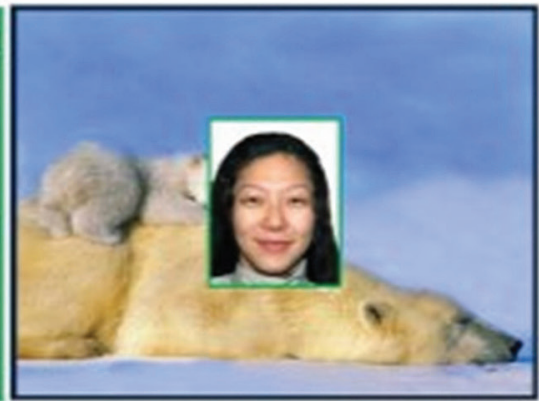

(1) Congruent

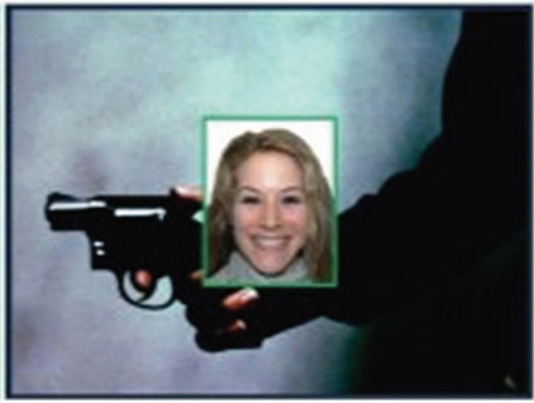

(2) Incongruent
(C) Attention

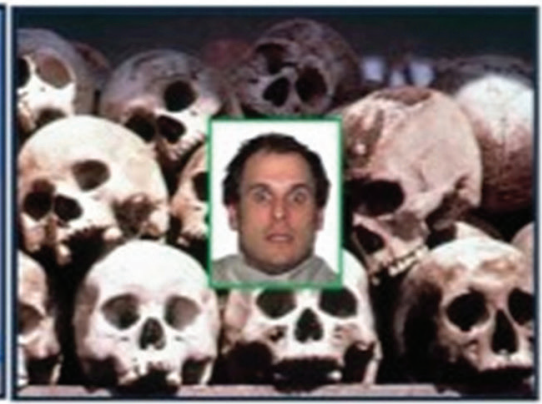

(1) Low

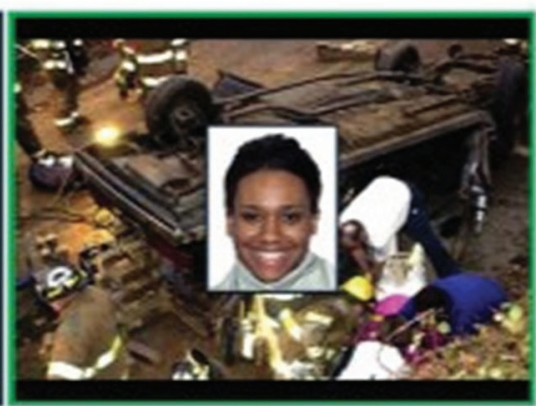

(2) High

Fig. 1: The Variable Affective Attention Task. Participants were asked to label the image framed in green as pleasant or unpleasant. The valence contrast (positive v. negative), congruency contrast (congruent v. incongruent) and attention contrast (low attention [face] v. high attention [scenes]) are displayed.

(participants had to focus on background scenes) or low (participants had to focus on the central facial stimuli). The time frame of the VAAT is described in Appendix 1. Participants were trained on the task outside the scanner.

We tested for differences between the task conditions of each contrast (i.e., valence, congruency and attention), differences between groups and group $\times$ task condition interactions for the behavioural measures of the VAAT (i.e., correct responses and reaction times). We used linear mixed models and repeated measures for each participant. We modelled the repeated measures as "intercept-only" participantspecific first-level random factors. Such models are more powerful than repeated-measures analysis of variance because they can account for missing values at a given time point without excluding the observation. We ran 6 models ( 3 contrasts and 2 behavioural measures; Appendix 1).

\section{Brain imaging}

\section{MRI acquisition}

We acquired all images on a 3 T Magnetom Verio MRI scanner (Siemens Healthineers) running VB17. After an initial localizing scan, we acquired functional data using a $T_{2}{ }^{*}$ - weighted gradient echo planar imaging sequence (repetition time $3000 \mathrm{~ms}$; echo time $30 \mathrm{~ms}$; field of view $192 \times 192 \mathrm{~mm}^{2}$; matrix $64 \times 64$; flip angle $90^{\circ}$; voxel size $\left.3 \times 3 \times 3 \mathrm{~mm}^{3}\right)$. We acquired 4 functional runs of 45 interleaved axial slices along the anterior-posterior commissure plane, with a continuous slice thickness of $3 \mathrm{~mm}$.

After the functional MRI scans, we acquired high-resolution anatomic images for anatomic identification using a sagittal, $T_{1}$-weighted, magnetization-prepared rapid gradient echo sequence (repetition time $1900 \mathrm{~ms}$; inversion time $900 \mathrm{~ms}$; echo time $2.26 \mathrm{~ms}$; flip angle $9^{\circ}$; parallel imaging with GRAPPA 2; field of view $256 \times 256 \mathrm{~mm}^{2}$; 176 slabs; voxel size $\left.1 \times 1 \times 1 \mathrm{~mm}^{3}\right)$.

We acquired diffusion-weighted images with an echo planar imaging sequence (30 directions; b-value $1000 \mathrm{~s} / \mathrm{mm}^{2}$; repetition time $11000 \mathrm{~ms}$; echo time $99 \mathrm{~ms}$; field of view $256 \times$ $256 \mathrm{~mm}^{2} ; 60$ slices; voxel size $2 \times 2 \times 2 \mathrm{~mm}^{3}$ )

Functional processing and statistical plan

Functional activity analysis

We analyzed all data using SPM8 software. ${ }^{34}$ We corrected raw activation maps for slice timing and motion correction, 
coregistered to the corresponding anatomic volume, normalized to the Montreal Neurological Institute standard space and smoothed with a $6 \mathrm{~mm}$ Gaussian kernel (details are provided in Appendix 1). For each participant, we calculated first-level contrast images to estimate blood-oxygenationlevel-dependent (BOLD) signal changes due to variations in valence (negative or positive), congruency (incongruent or congruent) and attention (attention to the scene [high] or attention to the face [low]). Then, we entered the first-level contrast images into second-level 2-sample $t$ tests with a random-effects statistical model to examine between-group effects. To test for associations between BOLD signal change and the AES, we performed voxel-wise linear regressions in the MDD group, where AES was the variable of interest, controlling for MADRS score. We used a region-of-interest (ROI) approach, focusing on the amygdala (valence contrast), dlPFC (attention contrast) and ACC (congruency contrast) ${ }^{19}$ as small-volume-correction ROIs, defined a priori (bilateral regions from the Automated Anatomic Labelling implemented in the WFU PickAtlas ${ }^{35}$ bound together), resulting in 3 independent masks (Appendix 1, Figure S1). Results were considered significant at a voxel-wise threshold of $p<$ 0.05 (small-volume corrected [SVC], family-wise error [FWE] corrected).

\section{Functional connectivity analyses}

We performed generalized psychophysiological interaction analyses (gPPI), which allowed us to study task-dependent functional connectivity in more than 2 task conditions. ${ }^{36}$ The parameter estimated using gPPI can be interpreted as the condition-specific functional connectivity of the seed region with the target region. We conducted separate gPPI analyses using each ROI (right and left anatomic regions [amygdala, ACC and dlPFC] bound together, resulting in 3 independent masks) as a seed region. We used this to examine variations in functional interactions according to valence (amygdala), congruency (ACC) or attention (dlPFC). For each participant, we created the seed masks using a $5 \mathrm{~mm}$ radius sphere ( $3 \mathrm{~mm}$ for the amygdala) around the coordinates of the participant-specific local maximums found (1) within the corresponding ROI of each contrast and (2) within $15 \mathrm{~mm}(10 \mathrm{~mm}$ for the amygdala) of the maximums of the healthy controls. ${ }^{722-25}$ This approach accounted for interindividual variability and is thought to be the most sensitive. ${ }^{37}$

Within each seed region, we calculated the physiologic variable by deconvolving and mean-correcting the first eigenvariate of the high-pass-filtered (128 s) BOLD signal after regressing out motion parameters. We computed psychophysiological interaction terms as the cross-product of the physiological variable and each task regressor (negative valence, positive valence, congruent, incongruent, low attentional load, high attentional load). Finally, we entered the physiological variable of the seed region, the psychological regressors and the psychophysiological interaction variables as regressors in a firstlevel generalized linear model. We also included the regressors from the artifact detection tool (Appendix 1) in the model as nuisance variables. We used the individual contrast images in second-level analyses using 2-sample $t$ tests for between-group differences. In addition to participant-specific seeds, we used the previously mentioned anatomic ROI seeds to describe between-group functional connectivity (Appendix 1, Figures S4, S5 and S6 and Table S2). Results were considered significant at a voxel-wise threshold of $p_{\mathrm{SVC}, \mathrm{FWE}}<0.05$, using the masks previously described.

\section{Relationship between apathy and functional connectivity}

We entered the individual contrast images testing for an effect of the psychophysiological interaction regressor into second-level multiple regressions for the dlPFC (attention contrast) as the seed ROI. We applied voxel-wise $t$ tests to identify voxels whose task-related changes in connectivity with the seed region were correlated with AES score (controlling for MADRS). We used the same statistical thresholds as described above.

\section{Preprocessing of diffusion-weighted images}

We used Brain Extraction Tool ${ }^{38}$ and ExploreDTI ${ }^{39}$ to preprocess the diffusion-weighted images and estimate the tensor. After creating a matrix that incorporated the $b$ values and $\mathrm{b}$ vectors, ExploreDTI applied motion- and eddy-currentinduced geometrical distortion corrections using an appropriate B-matrix rotation. ${ }^{40}$ The tensor was then estimated on a voxel basis using a linear least squares approach embedded in the informed Robust Extraction of kurtosis INDices with linear estimation (REKINDLE) algorithm, which is effective in the presence of physiologic noise. ${ }^{41}$ The resulting fractional anisotropy maps were warped into a common skeletonized space using tract-based spatial statistics algorithms to avoid partial-volume effects and smoothing estimation (defined a priori) ${ }^{24}$ from FSL (version 6.0). ${ }^{42}$ The full preprocessing procedure is described in Appendix 1.

\section{Tract-based spatial statistics}

We first compared the 2 groups (MDD and healthy controls) using a generalized linear model (2 groups unpaired option), accounting for age (de-meaned across groups). We also tested for positive and negative correlations between wholeskeleton voxel-wise fractional anisotropy values and AES scores within the MDD group, accounting for age and MADRS score. To do this, we used a single-group framework generalized linear model, accounting for the mean of the group in the model (i.e., intercept). For both hypotheses, we obtained statistical inference using the "randomize" tool, ${ }^{43}$ running 5000 permutations and using voxel-wise thresholdfree cluster enhancement (which integrates both the heights and spatial extents of clusters ${ }^{44}$ ) and $t$ statistics under the null hypothesis. We derived $p$ values from the proportions of the maximum statistics (i.e., threshold-free cluster enhancement or $t$ values) above the observed statistics at the voxel level. The type 1 error rate was set at $p_{\mathrm{FWE}}<0.05$ (whole-brain corrected) to correct for multiple comparisons across space. We did not use variance smoothing. We identified tracts using the Johns Hopkins University atlas $81 .^{45}$ 


\section{Results}

\section{General cognition and executive function}

Table 1 shows between-group differences and impairments on initiating processes (verbal fluencies) and attention (digit symbol subtest of the Wechsler Adult Intelligence Scale).

\section{Group comparisons}

\section{VAAT}

In the congruency contrast, participants with MDD made more errors than controls $(\beta=-0.79,95 \%$ confidence interval [CI] -1.35 to $-0.22 ; Z=-2.8 ; p<0.001)$, especially during incongruent trials ( $\beta=0.38,95 \%$ CI 0.14 to $0.62 ; Z=$ $3.1 ; p<0.001)$. Participants with MDD were slower than controls ( $\beta=243,95 \%$ CI 55.6 to $430.4 ; t_{47.8}=2.5 ; p=0.01$ ). We did not find a significant group $\times$ congruency interaction for reaction time $(\beta=-2.2,95 \% \mathrm{CI}-36.7$ to -32.3 ; $t_{12493}=-0.125 ; p=0.9$ ).

In the attention contrast, participants made more errors in the scene condition than in the face condition $(\beta=0.55,95 \%$ CI 0.37 to $0.74 ; Z=5.8 ; p<0.001$ ), and we found a significant group $\times$ attention interaction $(\beta=-0.49,95 \% \mathrm{CI}-0.7$ to -0.26 ; $Z=-4.1 ; p<0.001)$, suggesting that participants with MDD made more errors during the face trials. All participants were slower in the scene condition $(\beta=-335,95 \% \mathrm{CI}-359$ to $\left.-311.1 ; t_{12493}=-27.4 ; p<0.001\right)$ and participants with MDD were slower than controls $\left(\beta=214.4,95 \%\right.$ CI $27.1-401.7 ; t_{47.7}=$ $2.2 ; p=0.03)$, especially during face trials $(\beta=55.1,95 \% \mathrm{CI}$ 22.2 to $\left.88 ; t_{12493}=3.3 ; p<0.001\right)$.

In the valence contrast, all participants made more errors in the negative condition than in the positive condition $(\beta=$ $0.85,95 \%$ CI $0.65-1 ; Z=8.6 ; p<0.001)$. A significant group $\times$ valence interaction $(\beta=0.81,95 \% \mathrm{CI}-1$ to $-0.58 ; Z=-6.7 ; p<$ 0.001 ) suggested that participants with MDD made more errors during the positive trials. All participants were slower in the negative condition ( $\beta=-121.9,95 \% \mathrm{CI}-147$ to -96.8 ; $\left.t_{12493}=-9.5 ; p<0.001\right)$, and participants with MDD were slower than controls $\left(\beta=205.9,95 \%\right.$ CI 18.6 to $393.3 ; t_{47.8}=2.1$; $p=0.04)$, especially during positive trials $(\beta=71.9,95 \% \mathrm{CI}$ 37.5 to $\left.106.4 ; t_{12493}=4.1 ; p<0.001\right)$. All descriptive statistics are available in Appendix 1.

\section{Functional MRI}

Functional activity analyses

Between-group comparisons did not reveal significant differences in functional activity that survived correction for multiple testing, using an ROI approach or whole-brain analysis.

Functional connectivity analyses

Table 2 and Figure 2 show between-group significant differences in functional connectivity. We found no significant group differences in functional connectivity when

\begin{tabular}{|c|c|c|c|c|}
\hline Characteristic & $\begin{array}{c}\text { MDD } \\
(n=30)\end{array}$ & $\begin{array}{c}\mathrm{HC} \\
(n=25)\end{array}$ & $t_{54}$ & $p$ value \\
\hline Age & $47.57 \pm 11$ & $46.73 \pm 10.4$ & 0.3 & 0.76 \\
\hline Socioeconomic status & $13.3 \pm 2.4$ & $13.1 \pm 2.2$ & 0.3 & 0.78 \\
\hline Previous MDD episodes, $n$ & $4.6 \pm 3.5$ & - & - & - \\
\hline MDRS score & $137 \pm 8.1$ & $142.2 \pm 1.4$ & -3.4 & 0.001 \\
\hline Phonemic fluency, number of words & $16.7 \pm 7.1$ & $24.1 \pm 6.8$ & -4 & $<0.001$ \\
\hline Semantic fluency, number of words & $24.6 \pm 9.1$ & $32.4 \pm 7.4$ & -3.5 & 0.001 \\
\hline Action verbs fluency, number of words & $12 \pm 4.6$ & $15 \pm 5.3$ & -2.1 & 0.04 \\
\hline Stroop reading, number of words, corrected & $88.5 \pm 18.3$ & $105.4 \pm 10$ & -4 & $<0.001$ \\
\hline Stroop denomination, number of words, corrected & $66.1 \pm 16$ & $75.7 \pm 9.1$ & -2.6 & 0.01 \\
\hline Stroop interference, number of words & $34.5 \pm 15$ & $43.6 \pm 8.2$ & -2.6 & 0.01 \\
\hline Stroop interference score & $-2.3 \pm 8.4$ & $0.2 \pm 7.6$ & -1.1 & 0.3 \\
\hline Trail-Making Test A, s & $50 \pm 27.3$ & $36 \pm 13.7$ & 2.3 & 0.03 \\
\hline Trail-Making Test B, s & $111.1 \pm 62.4$ & $69.3 \pm 25.9$ & 3 & 0.004 \\
\hline Trail-Making Test B-A, s & $66.1 \pm 51.1$ & $38.4 \pm 22.4$ & 2.5 & 0.02 \\
\hline Modified Wisconsin Card Sorting Test, s & $196.6 \pm 68.2$ & $132.3 \pm 50.3$ & 3.8 & $<0.001$ \\
\hline Modified Wisconsin Card Sorting Test categories & $5.6 \pm 1.4$ & $6 \pm 0$ & -1.5 & 0.14 \\
\hline Modified Wisconsin Card Sorting Test errors & $3.5 \pm 5.3$ & $2.3 \pm 7.5$ & 0.6 & 0.5 \\
\hline Modified Wisconsin Card Sorting Test perseverative & $0.9 \pm 1.9$ & $0.1 \pm 0.4$ & 1.8 & 0.07 \\
\hline Digit symbol subtest (Wechsler Adult Intelligence Scale) & $55.9 \pm 14.5$ & $79.4 \pm 12.7$ & -6 & $<0.001$ \\
\hline Direct span & $5.4 \pm 1.1$ & $6 \pm 1$ & -2.1 & 0.04 \\
\hline Indirect span & $3.9 \pm 1.2$ & $4.6 \pm 1$ & -2.4 & 0.02 \\
\hline
\end{tabular}

$\mathrm{HC}=$ healthy controls; MDD = major depressive disorder; MDRS = Mattis Dementia Rating Scale; Stroop = Stroop Colour and Word Test

${ }^{*}$ Matched for socioeconomic status and age.

†Values are presented as mean \pm standard deviation. 
Table 2: Reduced and increased connectivity between the ACC, dIPFC and amygdala in patients with MDD compared with healthy controls

\begin{tabular}{|c|c|c|c|c|c|c|c|c|c|}
\hline Contrast & $\begin{array}{l}\text { Seed } \\
\text { region }\end{array}$ & $\begin{array}{c}\text { Group } \\
\text { differences }\end{array}$ & $\begin{array}{l}\text { Anatomic } \\
\text { region }\end{array}$ & $\begin{array}{l}\text { Brodmann } \\
\text { area }\end{array}$ & $\begin{array}{c}\text { MNI } \\
\text { coordinates, } \\
x, y, z\end{array}$ & $\begin{array}{l}\text { Cluster } \\
\text { size, } \\
\text { mL }\end{array}$ & $t_{54}$ & $\begin{array}{c}\text { Cluster } \\
p_{\mathrm{FWE}}{ }^{*}\end{array}$ & $\begin{array}{l}\text { Voxel } \\
p_{\mathrm{FWE}}{ }^{*}\end{array}$ \\
\hline $\begin{array}{l}\text { Negative > positive } \\
\text { valence }\end{array}$ & Left amygdala & $\mathrm{HC}>\mathrm{MDD}$ & Left ACC & 32 & $-6,46,0$ & 2.43 & 4.18 & 0.001 & 0.035 \\
\hline High > low attention & Right dIPFC & $\mathrm{HC}>\mathrm{MDD}$ & Right ACC & $9 \dagger$ & $14,46,28$ & 1.2 & 4.06 & 0.08 & 0.021 \\
\hline High > low attention & Right dIPFC & MDD > HC & Right amygdala & 36 & $26,6,-28$ & 0.35 & 3.35 & 0.13 & 0.048 \\
\hline
\end{tabular}

$\mathrm{ACC}=$ anterior cingulate cortex; dIPFC = dorsolateral prefrontal cortex; FWE = family-wise error; $\mathrm{HC}=$ healthy controls; $\mathrm{MDD}=$ major depressive disorder; $\mathrm{MNI}=\mathrm{Montreal}$ Neurological Institute.

${ }^{*}$ Cluster and voxel $p_{\mathrm{FWE}}$ values were obtained using the anatomic region of interest, derived from anatomic atlas labelling as a small-volume correction.

†Extending to the right dorsal ACC



Fig. 2: Functional coupling differences between the MDD $(n=30)$ and healthy control $(n=25)$ groups. (A) Reduced connectivity in MDD compared with healthy controls between seed regions in the left amygdala and the left ACC. (B) Reduced connectivity in MDD compared with healthy controls between seed regions in the right dIPFC and the right dACC. (C) Increased connectivity in MDD compared with healthy controls between seed regions in the right dIPFC and the right amygdala. Colour bars display the maximum $t$ values. The statistical threshold was set at $p<0.05$, small-volume (i.e., anatomic ROI) FWE-corrected. Clusters are overlaid on a single $T_{1}$ volume provided by SPM8. Montreal Neurological Institute slices are displayed. Bottom row: Box plots display the mean $\beta$ values extracted in the clusters (MarsBaR; http://marsbar.sourceforge.net/) from the first-level GLM PPI analysis. Error bars are standard deviations. ACC = anterior cingulate cortex; $\mathrm{dACC}=$ dorsal anterior cingulate cortex; dIPFC = dorsolateral prefrontal cortex; FWE = family-wise error; GLM = generalized linear model; $\mathrm{MDD}$ = major depressive disorder; $\mathrm{PPI}=$ psychophysiological interaction; $\mathrm{ROI}=$ region of interest.

using the ACC as the seed region during the congruent contrast. Whole-brain analyses revealed no differences that survived correction for multiple testing.

\section{Diffusion-weighted imaging}

We found decreased fractional anisotropy values in an extensive network ( $45.5 \mathrm{~mL})$; we observed the largest effect size in the anterior part of the cingulate cortex, the forceps minor and the uncinate (Appendix 1, Figure S4).

\section{Correlation with apathy scores}

\section{Functional results}

Functional activity analyses

We found a positive correlation between AES scores in participants with MDD and a BOLD signal increase in the ACC (peak -4, 50, $26[\mathrm{~mm}] ; t=4.08$; voxel $p_{\mathrm{FWE}}=0.014$; cluster $0.8 \mathrm{~mL}$; cluster $\left.p_{\mathrm{FWE}}=0.017\right)$ in the congruence contrast. 
Functional connectivity analyses

Regression analyses showed a positive correlation between AES scores and functional connectivity between the right dlPFC and the right ACC during the attention contrast. This indicated that the higher the apathy score, the stronger the coupling between the dIPFC and ACC, suggesting increased coactivation in attention- and effort-related structures when apathy is higher (Table 3 and Appendix 1, Figure S5) during processing of high-attention stimuli.

\section{Diffusion-weighted imaging}

Threshold-free cluster enhancement statistics revealed positive associations between AES scores and fractional anisotropy in 1 cluster $(0.9 \mathrm{~mL})$ located in the right posterior cerebellum. The $t$ statistics revealed 4 clusters located in the anterior and posterior parts of the cingulate cortex $(0.22 \mathrm{~mL}$ and $0.2 \mathrm{~mL}$, respectively) and the bilateral posterior part of the internal capsule (both $0.2 \mathrm{~mL}$; Figure 3).

\section{Discussion}

Using a task that allowed us to study emotional labelling in various conditions of attention and conflict, we showed that depression is associated with decreased functional connectivity between the left amygdala and the left perigenual ACC during the processing of negative stimuli.
Our results were consistent with the literature suggesting reduced functional connectivity between the rostral ACC and the amygdala in MDD using emotional tasks $\mathrm{s}^{6,7}$ or in the resting state. ${ }^{46,47}$

In healthy individuals, inflammation-induced mood degradation is associated with reduced functional activity between the subgenual ACC and the amygdala. ${ }^{48}$ Functional connectivity between the amygdala and the perigenual ACC was negatively associated with negative affect in controls. ${ }^{49}$ Carriers of the 5-HTTP short allele have reduced functional connectivity between the subgenual region and the amygdala during the perception of threatening faces, compared with carriers of the long allele. ${ }^{8}$

Reduced perigenual-amygdalar functional connectivity has been tested as an intermediate phenotype for at-risk individuals. However, first-degree relatives also show increased functional connectivity between the perigenual ACC and the amygdala compared with healthy controls and people with MDD, suggesting that this might be a mechanism for resilience. ${ }^{49}$ As well, in a sample of 12 women, increased effective connectivity has been reported between the left-sided subgenual ACC and the amygdala while implicitly processing dynamic fearful faces. ${ }^{50}$ Thus, both increased and decreased functional connectivity between the rostral ACC and the amygdala have been described. Beyond task designs and sample size and

Table 3: Positive correlation between AES score and functional coupling with the right ACC, using the right dIPFC as a seed region

\begin{tabular}{lcccccc}
\hline Contrast & Seed region & $\begin{array}{c}\text { Anatomic } \\
\text { region }\end{array}$ & $\begin{array}{c}\text { Brodmann } \\
\text { area }\end{array}$ & $\begin{array}{c}\text { MNI coordinates, } \\
x, y, z\end{array}$ & $\begin{array}{c}\text { Cluster } \\
\text { size, mL }\end{array}$ & Voxel $p_{\mathrm{FWE}}$ \\
\hline High $>$ low attention & Right dIPFC & Right ACC & 32 & $14,44,2$ & 1.05 & 5.2 \\
\hline ACC = anterior cingulate cortex; AES = Apathy Evaluation Scale; dIPFC = dorsolateral prefrontal cortex; FWE = family-wise error; MNI = Montreal Neurological Institute.
\end{tabular}

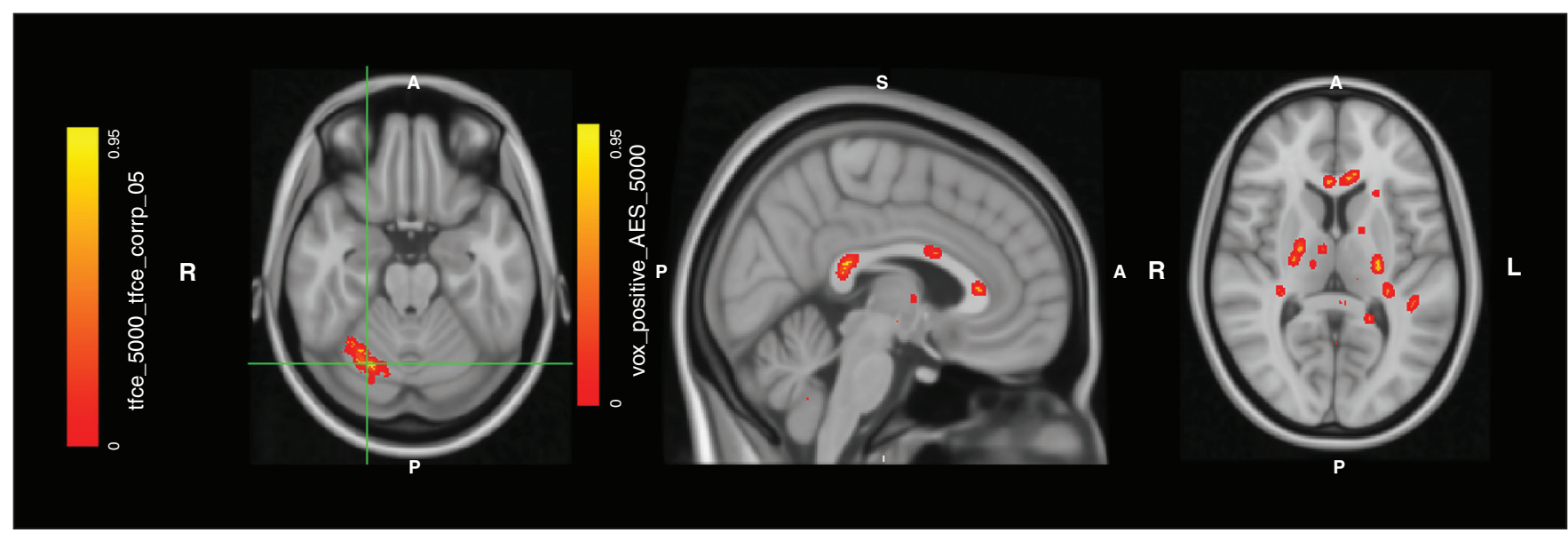

Fig. 3: Fractional anisotropy correlates of apathy. Left panel: positive correlation between AES scores and FA values in the right posterior cerebellum (Crus I) using voxel-wise TFCE statistics; 5000 permutations were performed. Statistical threshold was set at $p<0.05$, whole-brain FWE corrected. The colour bar displays $1-p$ values. Middle panel: the anterior and posterior cingulum. Right panel: the bilateral internal capsule. For both middle and right panel: voxel-wise $t$ statistics are used, 5000 permutations were performed. Statistical threshold was set at $p<0.05$, whole-brain FWE corrected. The colour bar displays $1-p$ values. Clusters are overlaid on a canonical template provided by fsleyes (https://fsl.fmrib.ox.ac.uk/fsl/fslwiki/FSLeyes; $1 \mathrm{~mm}$ resolution). AES = Apathy Evaluation Scale; FA = fractional anisotropy; FWE = family-wise error; TFCE = threshold-free cluster enhancement. 
characteristics, other modulating factors such as genotype, ${ }^{51} \mathrm{MDD}$ response, ${ }^{6} \mathrm{MDD}$ subtype ${ }^{47}$ and state or trait specificity ${ }^{47,49}$ could account for this variation.

We found decreased fractional anisotropy in an extensive network, including where the most significant difference was in the anterior cingulum and the forceps minor. ${ }^{26,27,52}$ This was consistent with previous results showing great variability in the findings of decreased fractional anisotropy. ${ }^{25,26,52-54}$ This variety has been associated with MDD clinical phenotype, ${ }^{53}$ MDD severity and duration, ${ }^{25,55}$ antidepressant intake ${ }^{54}$ and sex..$^{56}$ In the present study, the sample consisted of women with severe depression and impaired executive function, which might explain the size of the cluster for betweengroup differences.

When processing emotional stimuli that require high attention, participants with MDD showed increased functional connectivity between the right dIPFC and the right amygdala but reduced functional connectivity between the dIPFC and the dorsal ACC. Previous findings have shown that people with depression were unable to recruit frontal areas during cognitive control but had greater activation during an independent emotional task. ${ }^{57}$ Increased functional connectivity between the right dIPFC and the right amygdala could be due to an enhanced bottom-up emotional reactivity that prevents the recruitment of executive networks (such as the dlPFC and the dorsal ACC). Because gPPI cannot be used to infer the direction of the connectivity, another explanation could be that increased emotional reappraisal associated with high attention stimuli engages top-down control, as previously shown with the dlPFC. 5,57 In post-hoc analyses, we used tractography to test for associations between functional and structural connectivity (Appendix 1). We found a reduced number of tracts going through the right superior frontal gyrus to the right rostral ACC in participants with MDD compared to controls (mean \pm standard deviation $=$ $54.2 \pm 40$ v. $\left.83.7 \pm 48.8 ; t_{54}=0.02\right)$, corroborating our findings for functional connectivity. Reduced recruitment of structures engaged in the central executive network, including the dIPFC and the dorsal ACC, has been shown in MDD. ${ }^{57}$ In the present study, we have shown that this finding also stands for paradigms of emotional stimuli that require different degrees of attention within the same task.

Among participants with MDD, we found that apathy was associated with increased activation in the ACC and greater functional connectivity between the right dlPFC and right ACC. Apathy is a reduction in goal-directed activities and is thought to be supported by brain structures that belong to reward-processing and executive function. ${ }^{15}$ For example, empathy is associated with increased functional connectivity in the frontal cortices but also with the rest of the central executive network in mild cognitive impairment. ${ }^{22}$ In Parkinson disease, low symptoms of apathy are associated with increased functional connectivity between frontal areas and the caudate. ${ }^{23}$ Although these results cannot be directly compared with the present findings, they suggest adaptive functional strategies to compensate for goal-directed deficits. ${ }^{58}$

We found a positive association between apathy and fractional anisotropy in the anterior and posterior cingulum, posterior right cerebellum and bilateral internal capsule. In de novo Parkinson disease, the severity of apathy is associated with decreased fractional anisotropy in the ACC, forceps minor and posterior limb of the internal capsule part. ${ }^{59}$ However, people with Parkinson's disease and apathy had increased axial diffusivity in the anterior part of the cingulate cortex, extending to the uncinate and forceps minor. ${ }^{23}$ In MDD, anhedonia (closely related to apathy) is associated with fractional anisotropy in the anterior cingulum and the internal capsule. ${ }^{28}$ Using tractography, we could not find a positive association between AES scores and the number of tracts starting from the left superior frontal gyrus and the left rostral ACC $(r=-0.17, p=0.4)$ among participants with MDD, failing to reproduce our results for functional connectivity. We found increased fractional anisotropy in the posterior cerebellum, also involved in apathy in Parkinson disease. ${ }^{60}$ Moreover, converging findings from translational neuroscience provide evidence for involvement of the cerebellum in regulating high-order function such as emotion and motivation. ${ }^{61}$

These results require replication to further establish a network of apathy-related increased measures of fractional anisotropy, but they suggest that the anterior and posterior cingulum, posterior cerebellum and internal capsule might be regions of interest for characterizing apathy across disorders.

\section{Limitations}

For ethical reasons, participants with MDD were still taking their medications when they underwent MRI, but psychotropic drugs have been shown to modulate the BOLD signal, both in the resting state and during task-based echo planar imaging. ${ }^{62}$ However, our task-based design might have reduced this confounding bias by calculating firstlevel participant-based contrasts (i.e., participants were their own controls). However, we could rule out the possibility that psychotropic medications influenced BOLD signals differently when participants processed positive or negative stimuli. The various therapeutic combinations in the sample reduced sample homogeneity.

Four participants with MDD were treated with brain modulation. However, excluding these participants did not alter group comparisons at the behavioural level (Appendix 1, Tables S2, S3 and S4). This was also the case when we considered history of previous MDD episodes (mean \pm standard deviation $=5 \pm 3.5$; range $1-10$ ). We explored whether the number of previous MDD episodes affected the results, but we found no associations between number of episodes and fractional anisotropy or BOLD activation. Functional connectivity between the right dIPFC and amygdala were negatively associated with number of episodes $(\rho=-0.4 ; p=0.04)$. Other metrics of functional connectivity were not associated with number of episodes (Appendix 1).

Other functional connectivity techniques (such as dynamic causal modelling) would have enabled us to derive causality from seed-based time series with an a priori specified model, ${ }^{63}$ but this was not the case for the present study. 


\section{Conclusion}

We found that participants with MDD showed reduced functional connectivity between the amygdala and the perigenual ACC when they were processing negative stimuli. When they were processing high-attention stimuli, participants with MDD showed reduced functional connectivity between the dIPFC and the rostral ACC but increased functional connectivity between the dIPFC and the amygdala. Moreover, apathy was associated with increased functional connectivity between the dlPFC and the rostral ACC, as well as increased fractional anisotropy in the cingulum and the cerebellum. These results further strengthened our understanding of the underlying pathological process of emotion and cognitive interaction in MDD and its association with apathy. They also point to the ACC at the core of these processes and add to a growing literature targeting the ACC to alleviate emotional and motivational impairments in major depressive disorder.

Acknowledgments: The authors would like to thank Jacques Soulabaille, Stéphane Brousse and Odile Petton for scheduling the study and their help in gathering the healthy controls. This work is dedicated to the memory of Christian Barillot, director of the former Visages, now Empenn, Imaging research unit in Rennes from 2006 to 2020 .

Affiliations: From the EA 4712 Comportement et noyaux gris centraux, Université de Rennes 1, France (Robert, Batail, Drapier); the Psychiatry Department, Centre Hospitalier Guillaume Régnier, 108 Boulevard Général Leclerc, 35000, Rennes, France (Robert, Domain, Batail, Drapier); the Radiology Department, CHU Rennes, 2 Rue Henri le Guilloux, 35000 Rennes, France (Bannier, Ferre); the University of Rennes, CNRS, Inria, Inserm, IRISA UMR 6074, Empenn-ERL U 1228, 35000 Rennes, France (Bannier, Corouge, Ferre, Barillot); the Institut de Neurosciences de la Timone, Campus Santé Timone, 27, Bd Jean Moulin 13005 Marseille, France (Comte); the University of Lille \& CHU Lille, Inserm, U1171, Degenerative and Vascular Cognitive Disorders, 59000, Lille, France (Dondaine); and the Psychiatry Department, CHU Saint-Etienne, Team PsyR2-Centre de Recherche en Neuroscience de Lyon, (CRNL) CNRS UMR 5292-Inserm U1028, University of Lyon and Saint Etienne, France (Fakra).

Funding: This work is supported by the Fondation de l'Avenir (ET1628 ) and the Fondation Deniker. MRI data acquisition is supported by the Neurinfo MRI research facility from the University of Rennes I. Neurinfo is granted by the the European Union (FEDER), the French State, the Brittany Council, Rennes Metropole, Inria, Inserm and the University Hospital of Rennes.

Competing interests: G. Robert received funding for scientific talks from Janssen and Otsuka-Lundbeck (once each in the last 5 years). None of these were related to the current work. No other competing interests declared.

Contributors: G. Robert, E. Fakra and D. Drapier designed the study. G. Robert, E. Bannier, I. Courouge, T. Dondaine, J.-M. Batail and J.-C. Ferre acquired the data, which E. Bannier, M. Comte, L. Domain, E. Fakra and D. Drapier analyzed. G. Robert, E. Bannier, L. Domain and J.-C. Ferre wrote the article, which G. Robert, E. Bannier, M. Comte, L. Domain, I. Courouge, T. Dondaine, J.-M. Batail, E. Fakra and D. Drapier reviewed. All authors approved the final version to be published and can certify that no other individuals not listed as authors have made substantial contributions to the paper.

Content licence: This is an Open Access article distributed in accordance with the terms of the Creative Commons Attribution (CC BYNC-ND 4.0) licence, which permits use, distribution and reproduction in any medium, provided that the original publication is properly cited, the use is noncommercial (i.e., research or educational use), and no modifications or adaptations are made. See: https://creativecommons.org/licenses/by-nc-nd/4.0/

\section{References}

1. American Psychiatric Association. Diagnostic and statistical manual of mental disorders: DSM-5. 5th rev. ed. Washington (DC): American Psychiatric Publishing; 2013.

2. Nusslock R, Alloy LB. Reward processing and mood-related symptoms: an RDoC and translational neuroscience perspective. J Affect Disord 2017;216:3-16.

3. Fales CL, Barch DM, Rundle MM, et al. Altered emotional interference processing in affective and cognitive-control brain circuitry in major depression. Biol Psychiatry 2008;63:377-84.

4. Phillips ML, Drevets WC, Rauch SL, et al. Neurobiology of emotion perception II: implications for major psychiatric disorders. Biol Psychiatry 2003;54:515-28.

5. Erk S, Mikschl A, Stier S, et al. Acute and sustained effects of cognitive emotion regulation in major depression. J Neurosci 2010;30: 15726-34.

6. Vai B, Bulgarelli C, Godlewska BR, et al. Fronto-limbic effective connectivity as possible predictor of antidepressant response to SSRI administration. Eur Neuropsychopharmacol 2016;26:2000-10.

7. Musgrove DR, Eberly LE, Klimes-Dougan B, et al. Impaired bottom-up effective connectivity between amygdala and subgenual anterior cingulate cortex in unmedicated adolescents with major depression: results from a dynamic causal modeling analysis. Brain Connect 2015;5:608-19.

8. Pezawas L, Meyer-Lindenberg A, Drabant EM, et al. 5-HTTLPR polymorphism impacts human cingulate-amygdala interactions: a genetic susceptibility mechanism for depression. Nat Neurosci 2005;8:828-34

9. Wackerhagen C, Wüstenberg T, Mohnke S, et al. Influence of familial risk for depression on cortico-limbic connectivity during implicit emotional processing. Neuropsychopharmacology 2017;42:1729-38.

10. Batail JM, Palaric J, Guillery M, et al. Apathy and depression: which clinical specificities? Pers Med Psychiatry. 2018;7-8:21-6.

11. Robert $\mathrm{P}$, Lanctôt KL, Agüera-Ortiz L, et al. Is it time to revise the diagnostic criteria for apathy in brain disorders? The 2018 international consensus group. Eur Psychiatry 2018;54:71-6.

12. Fervaha G, Foussias G, Takeuchi $H$, et al. Motivational deficits in major depressive disorder: cross-sectional and longitudinal relationships with functional impairment and subjective well-being. Compr Psychiatry 2016;66:31-8.

13. Benoit M, Clairet S, Koulibaly PM, et al. Brain perfusion correlates of the apathy inventory dimensions of Alzheimer's disease. Int $J$ Geriatr Psychiatry 2004;19:864-9.

14. Robert GH, Le Jeune F, Lozachmeur C, et al. Preoperative factors of apathy in subthalamic stimulated Parkinson disease: a PET study. Neurology 2014;83:1620-6.

15. Le Heron C, Holroyd CB, Salamone J, et al. Brain mechanisms underlying apathy. J Neurol Neurosurg Psychiatry 2019;90:302-312.

16. Robert G, Le Jeune F, Dondaine T, et al. Apathy and impaired emotional facial recognition networks overlap in Parkinson's disease: a PET study with conjunction analyses. J Neurol Neurosurg Psychiatry 2014;85:1153-8.

17. Dondaine T, Philippot $\mathrm{P}$, Batail J-M, et al. Apathy alters emotional arousal in chronic schizophrenia. J Psychiatry Neurosci 2018;43: 170172.

18. Comte M, Cancel A, Coull JT, et al. Effect of trait anxiety on prefrontal control mechanisms during emotional conflict. Hum Brain Mapp 2015;36:2207-14.

19. Comte M, Schön D, Coull JT, et al. Dissociating bottom-up and top-down mechanisms in the cortico-limbic system during emotion processing. Cereb Cortex 2016;26:144-55.

20. Comte M, Zendjidjian XY, Coull JT, et al. Impaired cortico-limbic functional connectivity in schizophrenia patients during emotion processing. Soc Cogn Affect Neurosci 201813:381-90.

21. Cancel A, Comte M, Boutet C, et al. Childhood trauma and emotional processing circuits in schizophrenia: a functional connectivity study. Schizophr Res 2017;184:69-72.

22. Joo SH, Lee CU, Lim HK. Apathy and intrinsic functional connectivity networks in amnestic mild cognitive impairment. Neuropsychiatr Dis Treat 2016;13:61-7. 
23. Lucas-Jiménez O, Ojeda N, Peña J, et al. Apathy and brain alterations in Parkinson's disease: a multimodal imaging study. Ann Clin Transl Neurol 2018;5:803-14.

24. Smith SM, Jenkinson M, Johansen-Berg H, et al. Tract-based spatial statistics: voxelwise analysis of multi-subject diffusion data. Neuroimage 2006;31:1487-505.

25. Chen G, Hu X, Li L, et al. Disorganization of white matter architecture in major depressive disorder: a meta-analysis of diffusion tensor imaging with tract-based spatial statistics. Sci Rep 2016;6:21825.

26. Liao $\mathrm{Y}$, Huang $\mathrm{X}, \mathrm{Wu} \mathrm{Q}$, et al. Is depression a disconnection syndrome? Meta-analysis of diffusion tensor imaging studies in patients with MDD. J Psychiatry Neurosci 2013;38:49-56.

27. Zhang A, Leow A, Ajilore O, et al. Quantitative tract-specific measures of uncinate and cingulum in major depression using diffusion tensor imaging. Neuropsychopharmacology 2012;37:959-67.

28. Dillon DG, Gonenc A, Belleau E, et al. Depression is associated with dimensional and categorical effects on white matter pathways. Depress Anxiety 2018;35:440-7.

29. Zhang $\mathrm{Y}, \mathrm{Wu} \mathrm{J}, \mathrm{Wu} \mathrm{W}$, et al. Reduction of white matter integrity correlates with apathy in Parkinson's disease. Int J Neurosci 2018;128:25-31.

30. Albert PR. Why is depression more prevalent in women? J Psychiatry Neurosci 2015;40:219-21.

31. Montgomery SA, Asberg M. A new depression scale designed to be sensitive to change. Br J Psychiatry 1979;134:382-89.

32. Marin RS, Biedrzycki RC, Firinciogullari S. Reliability and validity of the Apathy Evaluation Scale. Psychiatry Res 1991;38:143-62.

33. Bradley MM, Lang PJ. The International Affective Picture System (IAPS) in the study of emotion and attention. In: Handbook of emotion elicitation and assessment. New York: Oxford University Press; 2007: 29-46.

34. Friston $\mathrm{K}$, Holmes AP, Worsley KJ, et al. Statistical parametric maps in functional imaging: a general linear approach. Hum Brain Mapp 1994;2:189-210.

35. Maldjian JA, Laurienti PJ, Kraft RA, et al. An automated method for neuroanatomic and cytoarchitectonic atlas-based interrogation of fMRI data sets. Neuroimage 2003;19:1233-9.

36. McLaren DG, Ries ML, Xu G, et al. A generalized form of contextdependent psychophysiological interactions (gPPI): a comparison to standard approaches. Neuroimage 2012;61:1277-86.

37. O'Reilly JX, Woolrich MW, Behrens TEJ, et al. Tools of the trade: psychophysiological interactions and functional connectivity. Soc Cogn Affect Neurosci 2012;7:604-9.

38. Smith SM. Fast robust automated brain extraction. Hum Brain Mapp 2002;17:143-55.

39. Leemans A, Jeurissen B, Sijbers J, et al. ExploreDTI: a graphical toolbox for processing, analyzing, and visualizing diffusion MR data. In: Proceedings of the 17th annual meeting of the International Society of Magnetic Resonance Medicine; 2009; Apr. 18-24; Hawaii. Concord (CA): International Society of Magnetic Resonance Medicine; 2009: 3537.

40. Leemans A, Jones DK. The B-matrix must be rotated when correcting for subject motion in DTI data. Magn Reson Med 2009;61:1336-49.

41. Tax CMW, Otte WM, Viergever MA, et al. REKINDLE: robust extraction of kurtosis INDices with linear estimation. Magn Reson Med 2015;73:794-808.

42. Jenkinson M, Beckmann CF, Behrens TEJ, et al. FSL. Neuroimage 2012;62:782-90.

43. Winkler AM, Ridgway GR, Webster MA, et al. Permutation inference for the general linear model. Neuroimage 2014;92:381-97.
44. Smith SM, Nichols TE. Threshold-free cluster enhancement: addressing problems of smoothing, threshold dependence and localisation in cluster inference. Neuroimage 2009;44:83-98.

45. Wakana D, Jiang H, Nagae-Poetscher LM, et al. Fiber tract-based atlas of human white matter anatomy. Radiology 2004;230:77-87.

46. Lui S, Wu Q, Qiu L, et al. Resting-state functional connectivity in treatment-resistant depression. Am J Psychiatry 2011;168:642-8.

47. Workman CI, Lythe KE, McKie S, et al. Subgenual cingulateamygdala functional disconnection and vulnerability to melancholic depression. Neuropsychopharmacology 2016;41:2082-90.

48. Harrison NA, Brydon L, Walker C, et al. Inflammation causes mood changes through alterations in subgenual cingulate activity and mesolimbic connectivity. Biol Psychiatry 2009;66:407-14.

49. Wackerhagen C, Veer IM, Erk S, et al. Amygdala functional connectivity in major depression-disentangling markers of pathology, risk and resilience. Psychol Med 2020;50:2740-50.

50. de Almeida JRC, Kronhaus DM, Sibille EL, et al. Abnormal leftsided orbitomedial prefrontal cortical-amygdala connectivity during happy and fear face processing: a potential neural mechanism of female MDD. Front Psychiatry 2011;2:69.

51. Costafreda SG, McCann P, Saker P, et al. Modulation of amygdala response and connectivity in depression by serotonin transporter polymorphism and diagnosis. J Affect Disord 2013;150:96-103.

52. Jiang J, Zhao Y-J, Hu X-Y, et al. Microstructural brain abnormalities in medication-free patients with major depressive disorder: a systematic review and meta-analysis of diffusion tensor imaging. I Psychiatry Neurosci 2017;42:150-63.

53. Coloigner J, Batail J-M, Commowick O, et al. White matter abnormalities in depression: a categorical and phenotypic diffusion MRI study. Neuroimage Clin 2019;22:101710.

54. Choi KS, Holtzheimer PE, Franco AR, et al. Reconciling variable findings of white matter integrity in major depressive disorder. Neuropsychopharmacol 2014;39:1332-9.

55. de Diego-Adeliño J, Pires P, Gómez-Ansón B, et al. Microstructural white-matter abnormalities associated with treatment resistance, severity and duration of illness in major depression. Psychol Med 2014;44:1171-82

56. Lyon M, Welton T, Varda A, et al. Gender-specific structural abnormalities in major depressive disorder revealed by fixel-based analysis. Neuroimage Clin 2019;21:101668.

57. Stange JP, Bessette KL, Jenkins LM, et al. Attenuated intrinsic connectivity within cognitive control network among individuals with remitted depression: temporal stability and association with negative cognitive styles. Hum Brain Mapp 2017;38:2939-54.

58. Hillary FG, Roman CA, Venkatesan U, et al. Hyperconnectivity is a fundamental response to neurological disruption. Neuropsychology 2015:29:59-75.

59. Prange S, Metereau E, Maillet A, et al. Early limbic microstructural alterations in apathy and depression in de novo Parkinson's disease. Mov Disord 2019;34:1644-54.

60. Robert G, Le Jeune F, Lozachmeur C, et al. Apathy in patients with Parkinson disease without dementia or depression: a PET study. Neurology 2012;79:1155-60.

61. Bostan AC, Strick PL. The basal ganglia and the cerebellum: nodes in an integrated network. Nat Rev Neurosci 2018;19:338-350.

62. Wandschneider B, Koepp MJ. Pharmaco fMRI: Determining the functional anatomy of the effects of medication. Neuroimage Clin 2016;12:691-7.

63. Friston KJ. Functional and effective connectivity: a review. Brain Connect 2011;1:13-36. 